\title{
Check-list das Orchidaceae do estado de Mato Grosso do Sul, Brasil
}

\author{
Fábio de Barros', Climbiê Ferreira Hall', \\ Vespasiano Borges de Paiva Neto² \& João Aguiar Nogueira Batista ${ }^{3}$
}

\begin{abstract}
'Instituto de Botânica, Núcleo de Pesquisa Orquidário do Estado, Caixa Postal 68041, CEP 04045-972, São Paulo, SP, Brasil. fdebarros@terra.com.br 2Universidade Federal de Minas Gerais, Instituto de Ciências Biológicas, Departamento de Botânica, Av. Antônio Carlos 6627. CEP 31270-910, Belo Horizonte, MG, Brasil.

3Universidade Federal do Mato Grosso do Sul, Campus Universitário de Chapadão do Sul, CEP 79560-000, Chapadão do Sul, MS, Brasil.
\end{abstract}

Recebido em 27.IX.2014

Aceito em 15.VI.2016

DOI 10.21826/2446-8231201873s287

RESUMO - É apresentada uma listagem atualizada das orquídeas ocorrentes naturalmente no estado do Mato Grosso do Sul, bem como comentários sobre novas ocorrências e comparações com inventários anteriores. A lista apresentada traz 211 espécies em 88 gêneros, o que representa um aumento de aproximadamente $142 \%$ no número de espécies quando se compara com a Lista de Espécies da Flora do Brasil e de cerca de $29 \%$ quando se compara com a outra listagem disponível para o Mato Grosso do Sul. Das espécies listadas, 36 representam novas ocorrências para o estado.

Palavras-chave: flora, orquídeas, região Centro-Oeste

ABSTRACT - Checklist of the Orchidaceae from the state of Mato Grosso do Sul, Brazil. An updated list of orchid species from the state of Mato Grosso do Sul, Brazil is presented, as well as notes on new records, and comparison with earlier inventories. The presented list has 211 species belonging to 88 genera, which denotes an increase of about $142 \%$ in the number of species when compared to the List of Species of Brazilian Flora, and about $29 \%$ when compared to the other available listing for the state of Mato Grosso do Sul. From the listed species, 36 represent new records for the state.

Keywords: Floristics, orchids, Midwest region

\section{INTRODUÇÃO}

A família Orchidaceae é uma das maiores, se não a maior, do reino vegetal, englobando cerca de 25.000 espécies (Chase et al. 2003). Orquídeas ocorrem em todas as regiões vegetadas do globo, embora sua distribuição não seja homogênea, havendo uma forte predominância de espécies nas regiões tropicais e subtropicais. Cerca de $70 \%$ das espécies da família são epífitas e orquídeas com esse hábito são exclusivamente tropicais e subtropicais (Atwood 1986).

A flora brasileira é bastante rica em espécies de Orchidaceae, com mais de 2.500 espécies (Barros et al. 2015), o que representa cerca de $10 \%$ das espécies registradas para a família no mundo. Em nosso país, orquídeas ocorrem em todas as formações vegetais e até em áreas modificadas por ação humana, embora sejam claramente mais numerosas em regiões florestais, especialmente em Florestas Ombrófilas e regiões campestres de altitude. Especialmente ricas são as formações vegetais relacionadas com a Floresta Atlântica, com mais de 1.400 espécies e com a Floresta Amazônica, com mais de 700 espécies. Embora o cerrado não seja especialmente rico em orquídeas, o Bioma Cerrado rivaliza com a Amazônia em número de espécies (quase 700 espécies), principalmente em função dos Campos Rupestres e das Florestas Ciliares. Nas regiões campestres, como seria de se esperar, predominam as espécies terrícolas, enquanto nas florestas as epífitas são, em geral, muito mais numerosas.

É interessante notar que a família Orchidaceae tem grande importância florística, mas, de um modo geral, pouca importância em termos fitofisionômicos. Em nenhuma formação vegetal as orquídeas podem ser consideradas dominantes na fisionomia, mesmo quando o número de espécies presentes é bastante alto (e.g. Munhoz \& Felfili 2006, Boldrini et al. 2008, Munhoz \& Felfili 2008, Palma et al. 2008). A referida família é formada exclusivamente por plantas herbáceas, embora algumas poucas possam apresentar órgãos endurecidos e com aparência lenhosa (p. ex. Epistephium). A família pode ser reconhecida por um conjunto de características morfológicas: raízes com velame, caule muitas vezes dividido em rizoma e cauloma, cauloma comumente intumescido em pseudobulbo, flores trímeras, uma das pétalas modificada em labelo, ovário ínfero, geralmente unilocular, apenas uma antera fértil (na maioria das espécies), filete e estilete adnados em uma estrutura colunar, denominada coluna ou ginostêmio, antera geralmente opercular, pólen geralmente fundido em 
polínias, óvulos muito numerosos, e sementes minúsculas e muito numerosas, normalmente tunicadas. Nenhuma dessas características, isoladamente, pode ser considerada exclusiva da família, mas a combinação concomitante de algumas delas permite caracterizar uma orquídea. Ressaltese, no entanto, que dentro da família há exceções para quase todas essas características.

Ao se tentar realizar um inventário das orquídeas do Mato Grosso do Sul esbarra-se, inicialmente em um problema histórico. Como o estado só foi emancipado do Mato Grosso em 1977, o que é bem pouco tempo em termos históricos, todo o material de herbário e a literatura botânica anterior a esse ano refere-se apenas a "Mato Grosso". Desse modo, se não houver uma definição clara de município ou referência a algum ponto geográfico conhecido, é impossível ter certeza se a informação se refere a uma localidade hoje localizada no Mato Grosso ou no Mato Grosso do Sul. Esse problema fica evidente ao se consultar obras que seriam importantes para a compilação das espécies presentes no estado, como por exemplo, Pabst \& Dungs $(1975,1977)$ e Cribb (1998).

Hoehne (1951) apresentou uma extensa lista de exsicatas coletadas no Mato Grosso, parte das quais, proveniente de Municípios hoje pertencentes ao Mato Grosso do Sul. Para a família Orchidaceae são sete espécies - Catasetum fimbriatum (Morren) Lindl., Catasetum. saccatum Lindl. var. chrystianum (Rchb. f.) Mansf., Cyrtopodium saintlegerianum Rchb. f., Eulophia longifolia (H.B.K.) Schltr. (=Eulophia alta (L.) Fawcet \& Rendle), Galeandra coxinnensis Hoehne (=Galeandra beyrichii Rchb. f.), Oncidium jonesianum Rchb. f. (=Cohniella jonesiana (Rchb. f.) Christenson) e Oncidium macropetalum Lindl. (=Alatiglossum macropetalum (Lindl.) Baptista) - todas coletadas em Corumbá ou Coxim. Cribb (1998) apresentou uma listagem de espécies de orquídeas como parte do inventário da flora do Mato Grosso organizado por Dubs (1998). Nessa obra não foi considerada a separação entre Mato Grosso e Mato Grosso do Sul e, portanto, a flora desses dois estados foi tratada de maneira conjunta. $\mathrm{Na}$ listagem de orquídeas Cribb (1998) considerou 233 espécies, 42 das quais indicadas para municípios hoje pertencentes ao Mato Grosso do Sul. Há, no entanto, poucos inventários de orquídeas realizados especificamente para áreas delimitadas do Mato Grosso do Sul, após sua emancipação. Entre os inventários desse tipo estão um relatório apresentado por Pagotto \& Souza (2006) sobre a Biodiversidade do Complexo Aporé-Sucuriú, que lista 21 espécies de orquídeas para a região (sendo nove sem identificação até o nível de espécie), além do artigo de Rodrigues et al. (2009), que cita 21 espécies em 19 gêneros para uma área no Município de Corumbá. Um pouco mais recente é o artigo de Rech et al. (2011) sobre orquídeas de uma mata ciliar no município de Dourados, citando 17 espécies em 13 gêneros. Há ainda alguns resumos de congresso referentes a projetos em andamento em áreas dos municípios de Chapadão do Sul e Costa Rica (Bocchese et al. 2011, Paiva Neto et al. 2011a, b). Os táxons citados nesses artigos e resumos somam 50 espécies em 33 gêneros, se considerarmos apenas aqueles identificados até o nível de espécie, incluindo os marcados como affinis (aff.) ou a conferir (conf.). Já a Lista de Espécies da Flora do Brasil (Barros et al. 2015), lista 87 espécies para o Mato Grosso do Sul, em 44 gêneros. Bem recentemente, Ostetto (2015) publicou um livro versando sobre as orquídeas do Mato Grosso do Sul cuja listagem, sem considerar os híbridos naturais e os táxons não identificados até o nível específico (62 no total), abrange 163 espécies em74 gêneros.

O objetivo do presente trabalho é apresentar uma listagem atualizada das espécies de Orchidaceae nativas ocorrentes no Mato Grosso do Sul, tendo em vista os problemas abordados acima e as novidades que têm sido obtidas em coletas recentes no estado.

\section{Principais Grupos de Pesquisa}

Em relação especificamente às orquídeas, essa situação só vem sendo revertida, vagarosamente, com o desenvolvimento de alguns projetos recentes visando o seu inventário em diferentes regiões do estado. É o caso de projetos em andamento nos municípios de Chapadão do Sul, Costa Rica e Dourados, os dois primeiros vinculados à Universidade Federal do Mato Grosso do Sul, campus de Chapadão do Sul, com a colaboração do Instituto de Botânica (SP) e o terceiro sob a responsabilidade da Universidade Federal da Grande Dourados. Somam-se às coletas decorrentes desses projetos, aquelas realizadas sistematicamente, há vários anos, pelo Dr. Arnildo Pott e Ms. Vali Pott, provenientes especialmente da região do Pantanal Sul Matogrossense.

\section{MATERIAL E MÉTODOS}

Para elaboração da presente listagem das espécies ocorrentes no Mato Grosso do Sul, foram consultados os materiais depositados nos herbários CGMS, COR, CPAP e SP (acrônimos de acordo com Thiers 2012). Também foram consultados os seguintes materiais provenientes de coletas recentes, ainda não registradas em herbário: (a) coletas realizadas pelo Dr. Vespasiano Borges de Paiva Neto e seus colaboradores, provenientes dos municípios de Bonito, Cassilândia, Chapadão do Sul e Costa Rica, em fase de registro no herbário SP; e (b) coletas realizadas pelo Dr. Arnildo Pott e Ms. Vali Pott, provenientes de diferentes localidades do estado, a serem depositados no herbário CGMS. O número de exemplares examinados totalizou cerca de 500 exsicatas. Também foram analisadas as informações de coleções determinadas por especialistas em Orchidaceae, contidas em bancos de dados dos herbários BHCB, CPMA, ESA, FUEL, HCF, HSJRP, HUEFS, RB, MBM, NY, SP, UB, UEC e UPCB (acrônimos de acordo com Thiers 2012), obtidas pelo Sistema de Informações SpeciesLink (2012). Tal procedimento só foi adotado nos casos em que se considerou que os dados eram confiáveis. Por último, foram levados em consideração dados de literatura, em especial a obra de Hoehne (1951), e os 
artigos de Cribb (1998), Rodrigues et al. (2009), Rech et al. (2011), Bocchese et al. (2011) e Paiva Neto et al. (2011a, b), além da Lista de Espécies da Flora do Brasil (Barros et al. 2015).

Ao todo, foram contabilizadas cerca de 600 exsicatas de Orchidaceae, identificadas em nível de espécie. Para cada espécie foi selecionado um espécime para ser citado como material testemunha no checklist, dando prioridade às coleções pessoalmente analisadas e recentemente coletadas. Um mapa informativo de número de coletas por município foi elaborado no programa $\operatorname{ArcGis}{ }^{\circledR}$ a partir de shape files obtidos no site do Ministério do Meio Ambiente (2012) e de informações contidas em todas as coleções de Orchidaceae disponíveis para o Mato Grosso do Sul, mesmo aquelas não identificadas em nível de espécie.

\section{Principais Acervos}

Devido à localização das instituições nas quais estão presentes os grupos de pesquisa envolvidos no inventário de orquídeas em diferentes regiões do Mato Grosso do Sul, assim como as principais localidades de proveniência das coletas, grande parte do material botânico coletado no estado, ao menos no que diz respeito às orquídeas, está depositado nos seguintes herbários: (a) da Fundação Universidade Federal do Mato Grosso do Sul (CGMS); (b) da Universidade Federal de Mato Grosso do Sul (COR); (c) do Centro de Pesquisas Agropecuárias do Pantanal (CPAP) (ambos); (d) da Cidade Universitária de Dourados (DDMS); e (e) do Instituto de Botânica, de São Paulo (SP). O primeiro situa-se em Campo Grande, capital do estado; o segundo e o terceiro estão localizados na cidade de Corumbá, contando especialmente com importantes coleções da região do Pantanal; o quarto, situado na cidade de Dourados e o quinto, em São Paulo, possuem projetos focados em orquídeas realizados ou em desenvolvimento.

\section{RESULTADOS E DISCUSSÃO}

No quadro 1 é apresentada a lista compilada a partir das fontes mencionadas no item Material e Métodos. Ela mostra 211 espécies em 88 gêneros, números bem maiores do que aqueles apontados para o estado do Mato Grosso do Sul até o recentemente. A Lista de Espécies da Flora do Brasil (Barros et al. 2015), com suas 87 espécies em 44 gêneros para o estado, representava, até recentemente, a listagem mais completa disponível. O recém lançado livro de Ostetto (2015), tratando das orquídeas do estado do Mato Grosso do Sul, já representou um importante acréscimo em relação a essa lista, com suas 163 espécies em 74 gêneros, ou seja, um aumento de cerca de $87 \%$. Já a lista aqui apresentada, representa um acréscimo, em número de espécies, de aproximadamente $142 \%$ em comparação com Lista de Espécies da Flora do Brasil (Barros et al. 2015), e de pouco mais de $29 \%$ em comparação com a lista de Ostetto (2015). Além disso, deve-se ressaltar que 36 das espécies aqui listadas constituem novos registros de ocorrência para o MS.

Parte das espécies citadas para o Mato Grosso do Sul nos trabalhos de Cribb (1998), Rech et al. (2011), Bocchese et al. (2011) e Paiva Neto et al. (2011a, b) não estão listadas no quadro 1 . Algumas dessas citações representam simplesmente nomes diferentes para as mesmas espécies listadas no quadro 1. É o caso, por exemplo, de Acianthera aff. macuconensis (Barb. Rodr.) F. Barros (Rech et al. 2011), Brassavola aff. flagellaris Barb. Rodr. (Paiva Neto et al. 2011a, b), Brassavola tuberculata Rchb. f. (Cribb 1998), Catasetum barbatum (Lindl.) Lindl. (Paiva Neto et al. 2011a, b), Catasetum inconstans Hoehne, Cytopodium cf. palmifrons Rchb. f. \& Warm. (Paiva Neto et al. 2011b) e Encyclia linearifolioides (Kraenzl.) Hoehne (Bocchese et al. 2011) (Cribb 1998). O aparecimento de Lophiaris pumila (Lindl.) Braem (sin.: Oncidium pumilum Lindl.) nas listas (Cribb 1998, Bocchese et al. 2011, Rech et al. 2011) pode causar estranhesa, de início, por se tratar de uma espécie mais comum em áreas relacionadas com a Mata Atlântica, mas sua ocorrência já foi apontada também em áreas com predominância de Cerrado do Brasil Central, por exemplo, por Pellizzaro et al. (2004), para o DF (tratada como Oncidium pumilum). A ocorrência de Barbosella crassifolia (Edwall) Schltr., citada por Bocchese et al. (2011) é um pouco mais difícil de explicar, uma vez que é uma espécie mais comumente relacionada com a Mata Atlântica, e nunca encontramos nenhum material que pudesse atestar sua ocorrência no MS. A ocorrência de Dendrobium floribundum Rchb. f., espécie citada por Bocchese et al. (2011) se deve, certamente, a uma identificação equivocada, uma vez que o gênero Dendrobium é, basicamente, asiático, não tendo representantes nativos nas Américas. A julgar pela foto da planta interpretada como tal na página mantida pelo autor (Bocchese 2015) trata-se, na verdade, de Epidendrum densiflorum Lindl. A ocorrência de Encyclia leucantha Schltr. no Mato Grosso do Sul, citada por Cribb (1998) para o município de Aquidauana, é improvável; trata-se de uma espécie da Colômbia e Venezuela. Não encontramos nenhuma evidência, também, da ocorrência de Habenaria vaupellii Rchb. f. \& Warm. no estado, embora citada por Cribb (1998) para o municípío de Rio Brilhante; neste caso, no entanto, a ocorrência é possível, por ser uma espécie já encontrada em áreas de cerrado de Minas Gerais, São Paulo e Paraná, ou então, trata-se de identificação equivocada de Habenaria johannensis Barb.Rodr., espécie muito próxima. Finalmente devemos comentar a citação de Epidendrum aquaticum Lindl. e Galeandra coxinnensis Hoehne para o estado, por Cribb (1998); estes dois nomes foram considerados, aqui, como sinônimos, respectivamente, de Epidendrum dendrobioides Thunb. e Galeandra beyrichii Rchb.f.

A maioria das espécies da listagem de Ostetto (2015) também aparece na lista aqui apresentada, exceto alguns nomes, ou por serem considerados sinônimos de nomes que aparecem no Quadro 1, ou por terem sido considerados como nomes mal aplicados. Alguns exemplos do primeiro caso: Amblostoma amblostomoides (Hoehne) F.Barros [= Epidendrum amblostomoides Hoehne], Galeandra chapadensis Campacci [= Galeandra blanchetii E.S.Rand], 
Anacheilium marcilianum Campacci [= Prosthechea vespa (Vell.) W.E.Higgins], Anacheilium fragrans (Sw.) Acuña [= Prosthechea fragrans (Sw.) W.E.Higgins], Sarcoglottis gonzalezii L.C.Menezes [= S. curvisepala Szlach. \& Rutk.], e Laelia lundii (Rchb.f. \& Warm.) Rchb.f. \& Warm. [=Microlaelia lundii (Rchb.f.) Chiron \& V.P.Castro]. Alguns exemplos do segundo caso: Schomburgkia crispa Lindl. [aqui interpretada como Laelia gloriosa (Rchb.f.) L.O.Williams], Koellensteinia tricolor (Lindl.) Rchb.f. [aqui interpretada como Koellensteinia eburnea (Barb. Rodr.) Schltr.], Ornithocephalus myrticola Lindl. [aqui interpretada como Ornithocephalus cujeticola Barb.Rodr.] e Orleanesia mineirosensis Garay [aqui interpretada como Orleanesia yauaperyensis Barb.Rodr.].

O aumento de 87 espécies na Lista do Brasil, para 163 espécies na listagem de Ostetto (2015) e 211 na presente lista, mostra quão desconhecida ainda é a flora do Mato Grosso do Sul, o que está de acordo com afirmações de alguns botânicos, que consideram que o MS é um dos estados mais carentes de coletas no Brasil (e.g. GomesKlein \& Rizzo 2006). Uma comparação rápida do número de espécies e gêneros de orquídeas apontados na Lista de Espécies de Flora do Brasil (Barros et al. 2015), para os estados brasileiros que possuem áreas consideráveis de Cerrado (Tab. 1), mostra o Mato Grosso do Sul com números menores que Goiás, que possui área geográfica equivalente. Mas também menores, se comparados com os do Distrito Federal, que possui uma área geográfica cerca de 60 vezes menor. Isso indica que tais números devem estar refletindo a falta de coletas, mais do que a verdadeira composição florística do estado. Entre os estados considerados na tabela 1, situação semelhante à do Mato Grosso do Sul fica evidente em Tocantins.

\section{Principais Lacunas de Conhecimento}

Um aspecto a ressaltar é que a grande maioria dos materiais de herbário coletados no MS, assim como a maioria dos materiais citados na literatura especializada para esse estado, provém de poucos municípios, com predominância de Bonito, Chapadão do Sul, Costa Rica, Dourados e Corumbá. Portanto, pode-se afirmar que todo o restante do estado representa uma grande lacuna de conhecimento florístico para as orquídeas, como ilustrado na figura 1. Isso pode ser considerado um grande viés na representatividade do material coletado e indica a necessidade de se intensificar as coletas e inventários para os outros 75 municípios do MS, especialmente no sul e leste do estado.

Quanto à flora de orquídeas do estado, uma análise do Quadro 1 mostra uma mistura de elementos de diferentes Biomas, com clara predominância de espécies relacionadas com a Amazônia e o Cerrado, e menor representatividade de espécies relacionadas com a Mata Atlântica. Uma possível explicação é a falta de coletas na região sul do estado, onde predominam áreas de Mata Atlântica. No entanto, essa situação, percebida quando se analisa as orquídeas do estado como um todo, não se repete, necessariamente, em inventários locais. Rech et al. (2011), por exemplo, consideraram que há predominância de espécies da Floresta Estacional Semidecídua entre as orquídeas encontradas numa mata ciliar do rio Dourados, embora também tenham encontrado espécies de Floresta Ombrófila e Cerrado.

\section{Perspectivas de Pesquisa para o Grupo nos próximos 10 anos}

Uma possibilidade seria iniciar o inventário florístico pelas áreas de proteção ambiental localizadas, total ou parcialmente, dentro dos limites do estado. Uma consulta aos dados estatísticos do Mato Grosso do Sul (Mato Grosso do Sul 2009), mostra que o estado conta com várias áreas de proteção, as quais incluem unidades federais [Parque Nacional de Ilha Grande (compartilhado com o estado do Paraná); Parque Nacional da Serra da Bodoquena, Parque Nacional do Pantanal Matogrossense (compartilhado com o estado do Mato Grosso); Área de Proteção Ambiental Ilhas e Várzeas do Rio Paraná (compartilhada com os estados do Paraná e de São Paulo); Parque Nacional de Emas], estaduais [Parque Estadual da Serra de Sonora; Parque Estadual das Nascentes do Rio Taquari; Parque Estadual das Várzeas do Rio Ivinhema; Parque Estadual do Pantanal do Rio Negro; Parque Estadual do Prosa; Parque Estadual das Matas do Segredo; Área de Proteção Ambiental Rio

Tabela 1. Riqueza de orquídeas em estados brasileiros que possuem áreas consideráveis de Cerrado, com base na Lista de Espécies da Flora do Brasil (Barros et al. 2015), comparada com a riqueza no Mato Grosso do Sul, baseada nos números do presente trabalho.

\begin{tabular}{|c|c|c|c|}
\hline Região/Estado & Área do estado $\left(\mathrm{km}^{2}\right)$ & Número de gêneros & Número de espécies \\
\hline \multicolumn{4}{|l|}{ Região Centro-Oeste } \\
\hline Distrito Federal (DF) & $5.801,9$ & 80 & 252 \\
\hline Goiás (GO) & $340.086,7$ & 73 & 276 \\
\hline Mato Grosso (MT) & $903.357,9$ & 93 & 333 \\
\hline Mato Grosso do Sul (MS) & $358.158,7$ & 88 & 211 \\
\hline \multicolumn{4}{|l|}{ Região Sudeste } \\
\hline Minas Gerais (MG) & $586.528,3$ & 155 & 945 \\
\hline São Paulo (SP) & $248.209,4$ & 152 & 861 \\
\hline \multicolumn{4}{|l|}{ Região Nordeste } \\
\hline Bahia (BA) & $567.295,7$ & 140 & 557 \\
\hline \multicolumn{4}{|l|}{ Região Norte } \\
\hline Tocantins (TO) & $277.620,9$ & 44 & 123 \\
\hline
\end{tabular}




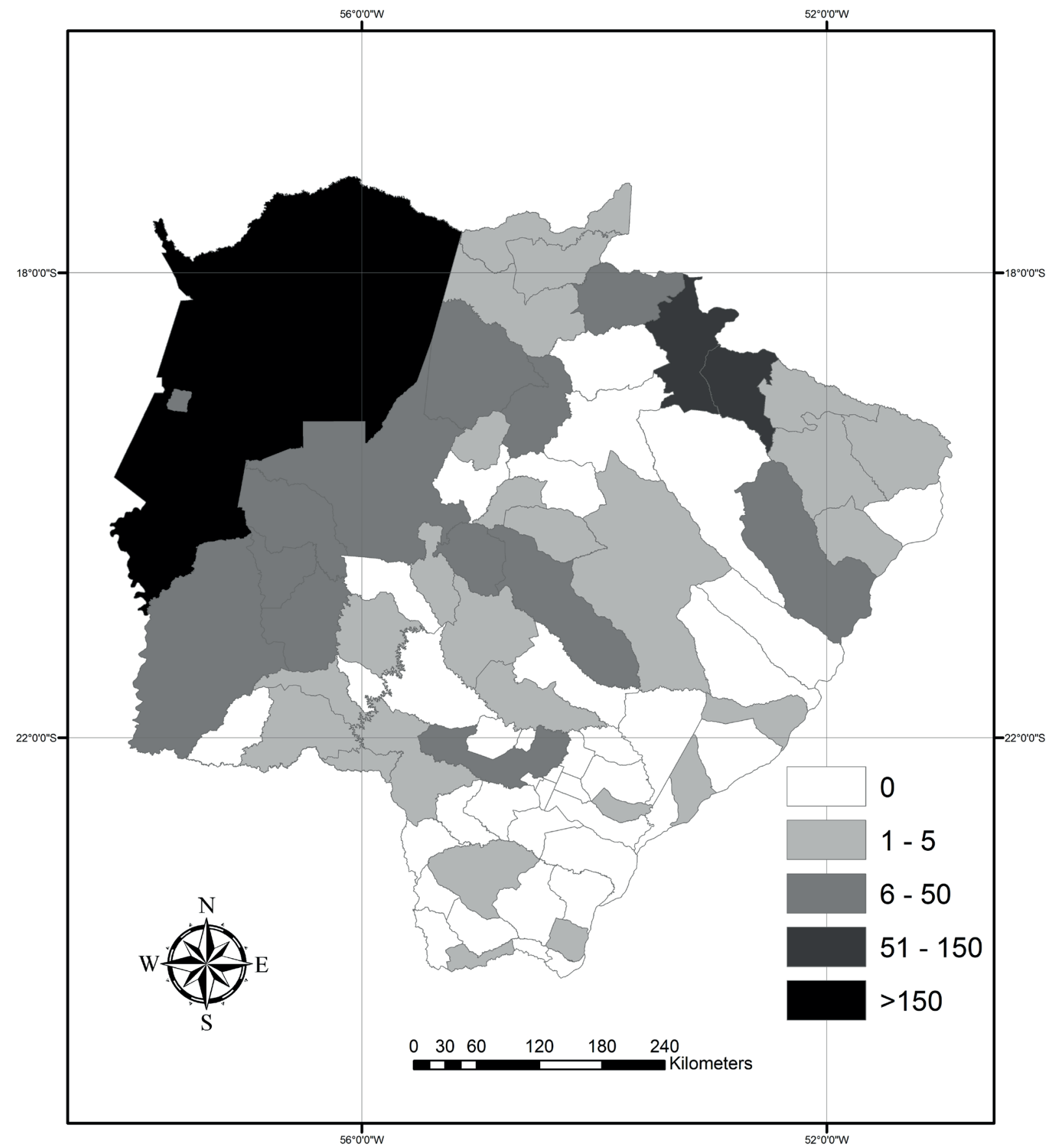

Fig. 1. Mapa do Mato Grosso do Sul contendo dados do número de coletas de espécimes de Orchidaceae em cada município.

Cênico Rotas Monçoeiras; Área de Proteção Ambiental Estrada Parque de Piraputanga] e municipais [p. ex. Área de Proteção Ambiental das Bacias do Rio Aporé e do Rio Sucuriu; Parque Natural Municipal de Piraputangas; Parque Natural Municipal do Córrego Cumandaí; Parque Natural Municipal do Paragem; etc., num total de quase 50], sem contar vários Monumentos Naturais e Reservas Particulares do Patrimônio Natural (RPPNs). Essas áreas de proteção totalizam cerca de 4.306.752,06 ha (Mato Grosso do Sul 2009) e abrangem quase todas as regiões do estado. Deve-se notar que, ao menos em relação à família Orchidaceae, há pouquíssimos materiais de herbário coletados nessas áreas.

\section{AGRADECIMENTOS}

Aos curadores dos herbários visitados, por permitirem o acesso ao material neles depositado e, em especial, ao Dr. Arnildo Pott e à Ms. Vali Pott, que facultaram o exame de coletas suas ainda não depositadas no herbário CGMS. Ao Ms. Augusto Francener Nogueira Gonzaga pelo auxílio na confecção do mapa. À Fundação de Amparo à Pesquisa do Estado de São Paulo, ao Conselho Nacional de Desenvolvimento Científico e Tecnológico e à Fundação de Apoio ao Desenvolvimento do Ensino, Ciência e Tecnologia do Estado de Mato Grosso do Sul pelas bolsas e auxílios recebidos pelos autores. 
Quadro 1. Lista das espécies de Orchidaceae ocorrentes no Estado do Mato Grosso do Sul, Brasil, com respectivos Voucher ou Referências Bibliográficas. NMS = novo registro para o Mato Grosso do Sul.

\begin{tabular}{|c|c|c|}
\hline Espécie & Voucher ou Referência Bibliográfica & Observações \\
\hline $\begin{array}{l}\text { Acianthera aphtosa (Lindl.) Pridgeon \& } \\
\text { M.W.Chase }\end{array}$ & Ostetto (2015) & \\
\hline A. luteola (Lind1.) Pridgeon \& M.W.Chase & Ostetto (2015) & \\
\hline A. pubescens (Lindl.) Pridgeon \& M.W.Chase & D.P. Rodriguez 2 (COR, SP) & \\
\hline A. ramosa (Barb.Rodr.) F.Barros & V.B. Paiva Neto 31 (SP) & NMS \\
\hline A. recurva (Lindl.) Pridgeon \& M.W.Chase & Rech et al. (2011) & \\
\hline $\begin{array}{l}\text { A. sonderiana (Rchb.f.) Pridgeon \& } \\
\text { M.W.Chase }\end{array}$ & Ostetto (2015) & \\
\hline Alatiglossum fuscopetalum (Hoehne) Baptista & V.B. Paiva Neto 93 (SP) & \\
\hline A. macropetalum (Lindl.) Baptista & D.P. Rodriguez 63 (COR, SP) & \\
\hline A. longipes (Lindl.) Baptista & Ostetto (2015) & \\
\hline $\begin{array}{l}\text { Anathallis brevipes (Focke) Pridgeon \& } \\
\text { M.W.Chase }\end{array}$ & M. Costa 01 (SP) & NMS \\
\hline $\begin{array}{l}\text { A. aff. imbricata (Barb. Rodr.) F.Barros \& } \\
\text { F.Pinheiro }\end{array}$ & V.B. Paiva Neto 72 (SP) & \\
\hline A. marginata (Barb. Rodr.) F. Barros \& Barberena & V.B. Paiva Neto 97 (SP) & NMS \\
\hline A. aff. aristulata (Lindl.) Luer & Rech et al. (2011) & $\begin{array}{l}\text { Citada por Rech et al. (2011) como A. aff. } \\
\text { montipelladensis (Hoehne) F. Barros }\end{array}$ \\
\hline A. obovata (Lindl.) Pridgeon \& M.W.Chase & Ostetto (2015) & \\
\hline Aspasia variegata Lindl. & F. Barros et al. 3180 (SP) & \\
\hline Aspidogyne foliosa (Poepp. \& Endl.) Garay & Ostetto (2015) & \\
\hline A. longicornu (Cogn.) Garay & D.P. Rodriguez 13 (COR, SP) & \\
\hline $\begin{array}{l}\text { Baptistonia cornigera (Lindl.) Chiron \& } \\
\text { V.P.Castro }\end{array}$ & Ostetto (2015) & \\
\hline B. lietzei (Regel) Chiron \& V.P.Castro & Ostetto (2015) & \\
\hline B. pubes (Lindl.) Chiron \& V.P.Castro & Ostetto (2015) & \\
\hline B. riograndensis (Cogn.) Chiron \& V.P.Castro & V.B. Paiva Neto 32 (SP) & NMS \\
\hline B. sarcodes (Lindl.) Chiron \& V.P.Castro & Ostetto (2015) & \\
\hline Bifrenaria tetragona (Lindl.) Schltr. & Ostetto (2015) & \\
\hline Bletia catenulata Ruiz \& Pav. & A. Penha et al. 379 (CGMS) & \\
\hline Brassavola cebolleta Rchb.f. & M.A Lima 01 (SP) & \\
\hline B. martiana Lindl. & Ostetto (2015) & \\
\hline B. rhomboglossa Pabst & H.G. Wiesberg s.n. (HB68928) & Holótipo \\
\hline Bulbophyllum epiphytum Barb. Rodr. & G. Hatschbach 47226 (CGMS, HUEFS, MGM) & \\
\hline B. insectiferum Barb. Rodr. & R. Antonelli \& A. G. Boaretto 02 (SP) & \\
\hline B. micranthum Barb. Rodr. & Rech et al. (2011) & \\
\hline B. tripetalum Lindl. & Rech et al. (2011) & \\
\hline Campylocentrum aromaticum Barb.Rodr. & V.B. Paiva Neto \& R. Antonelli 109 (SP) & NMS \\
\hline C. fasciola (Lindl.) Cogn. & V.B. Paiva Neto \& R. Antonelli 67 (SP) & \\
\hline C. grisebachii Cogn. & Ostetto (2015) & \\
\hline C. hasslerianum Hoehne & D.P. Rodriguez 8 (COR, SP) & \\
\hline C. micranthum (Lindl.) Rolfe. & Ostetto (2015) & \\
\hline C. neglectum (Rchb.f. \& Warm.) Cogn. & Ostetto (2015) & \\
\hline C. pachyrrhizum (Rchb.f.) Rolfe & V.B. Paiva Neto 70 (SP) & NMS \\
\hline C. robustum Cogn. & Ostetto (2015) & \\
\hline C. sellowii (Rchb. f.) Rolfe & R. Reiss $77(\mathrm{NY})$ & \\
\hline Capanemia micromera Barb.Rodr. & Rech et al. (2011) & \\
\hline C. superflua (Rchb.f.) Garay & Ostetto (2015) & \\
\hline Catasetum fimbriatum (Morren) Lindl. & V.J. Pott 1304 (CPAP) & \\
\hline C. lanciferum Lindl. & M.A. Lima 02 (SP) & \\
\hline C. longifolium Lindl. & M.A. Lima \& V.B. Paiva Neto 05 (SP) & \\
\hline C. osculatum Lacerda \& V.P.Castro & V.B. Paiva Neto 13 (SP) & \\
\hline C. rooseveltianum Hoehne & Ostetto (2015) & \\
\hline C. vinaceum (Hoehne) Hoehne & E.L.M. Catharino s.n. (SP170302) & $\begin{array}{l}\text { Parátipo de Catasetum taquariense } \\
\text { Bicalho, F. Barros \& Moutinho }\end{array}$ \\
\hline Cattleya nobilior Rchb.f. & D.P. Rodriguez 15 (COR) & \\
\hline C. walkeriana Gardner & A. Pott et al. 11687 (CGMS) & \\
\hline
\end{tabular}


Quadro 1. Cont.

\begin{tabular}{|c|c|c|}
\hline Espécie & Voucher ou Referência Bibliográfica & Observações \\
\hline Chaubardia surinamensis Rchb. f. & V.B. Paiva Neto 17 (SP) & \\
\hline Cleistes bella Rchb.f. \& Warm. & G. Hatschbach 35957 (MBM) & \\
\hline C. paranaensis (Barb.Rodr.) Schltr. & G. Hatschbach 35973 (MBM) & \\
\hline Cohniella cebolleta (Jacq.) Christenson & V.J. Pott 240 (CPAP, SP) & \\
\hline C. jonesiana (Rchb. f.) Christenson & I.M.Bortolotto et al. s.n. (COR5387, SP) & \\
\hline Coppensia bifolia (Sims) Dumort. & Ostetto (2015) & \\
\hline C. flexuosa (Lodd.) Campacci & Ostetto (2015) & \\
\hline C. hydrophila (Barb.Rodr.) Campacci & Ostetto (2015) & \\
\hline Corymborkis flava (Sw.) Kuntze & L.C. Rodrigues 97 (CGMS) & NMS \\
\hline Cyanaeorchis arundinae (Rchb. f.) Barb.Rodr. & G. Hatschbach 25107 (NY) & \\
\hline Cyclopogon congestus (Vell.) Hoehne & V.B. Paiva Neto 28 (SP) & \\
\hline C. elatus (Sw.) Schltr. & Ostetto (2015) & \\
\hline Cycnoches haagii Barb.Rodr. & V.B. Paiva Neto 68 (SP) & \\
\hline Cyrtopodium blanchetii Rchb.f. & G. Hatschbach \& C. Koczicki 33082 (MBM) & \\
\hline C. brandonianum Barb.Rodr. & Ostetto (2015) & \\
\hline C. fowliei L.C.Menezes & A. Seidel 1266 (HB) & NMS \\
\hline C. gonzalezii L.C.Menezes & Ostetto (2015) & \\
\hline C. hatschbachii Pabst & S.N. Moreira et al. 346 (CGMS) & \\
\hline C. paludicolum Hoehne & V.B. Paiva Neto \& M.A. Lima 23 (SP) & \\
\hline C. parviflorum Lindl. & A. Seidel 1161 (HB) & NMS \\
\hline C. poecilum Rchb.f. \& Warm. & Ostetto (2015) & \\
\hline C. saintlegerianum Rchb. f. & M.A. Lima 08 (SP) & \\
\hline C. sarneyanum L.C.Menezes & Ostetto (2015) & \\
\hline C. virescens Rchb.f. \& Warm. & G.A. Damasceno Junior et al. 2783 (COR) & \\
\hline Dichaea matogrossensis Brade & Ostetto (2015) & \\
\hline Dryadella zebrina (Porsch) Luer & V.B. Paiva Neto \& R. Antonelli 20 (SP) & NMS \\
\hline Encyclia argentinensis (Speg.) Hoehne & V.B. Paiva Neto \& N.R. Lenhard 98 (SP) & \\
\hline E. conchaechila (Barb.Rodr.) Porto \& Brade & M.A.C. Chaparro et al. 6 (COR, CPAP) & \\
\hline Epidendrum amblostomoides Hoehne & V.B. Paiva Neto \& R. Antonelli 85 (SP) & $\begin{array}{c}\text { Citada por Ostetto (2015) como } \\
\text { Amblostoma amblostomoides Hoehne) } \\
\text { F.Barros }\end{array}$ \\
\hline E. anceps Jacq. & V.B. Paiva Neto 82 (SP) & \\
\hline E. avicula Lindl. & Ostetto (2015) & \\
\hline E. cf. bahiense Rchb.f. & V.B. Paiva Neto \& R. Antonelli 61 (SP) & NMS \\
\hline E. campestre Lindl. & V.B. Paiva Neto 41 (SP) & \\
\hline E. coronatum Ruiz \& Pav. & $\begin{array}{l}\text { G.A. Damasceno Junior \& D.P. Rodriguez } 1986 \\
\text { (CGMS, SP) }\end{array}$ & \\
\hline E. dendrobioides Thunb. & V.B. Paiva Neto \& R. Antonelli 55 (SP) & \\
\hline E. densiflorum Lindl. & D.P. Rodriguez 64 (COR) & \\
\hline E. latilabre Lindl. & Ostetto (2015) & \\
\hline E. nocturnum Jacq. & F. Barros et al. 3175 (SP) & \\
\hline E. rigidum Jacq. & V.B. Paiva Neto 11 (SP) & \\
\hline E. secundum Jacq. & Ostetto (2015) & \\
\hline E. stiliferum Dressler & Ostetto (2015) & \\
\hline E. strobiliferum Rchb.f. & V.B. Paiva Neto 74 (SP) & NMS \\
\hline Epistephium lucidum Cogn. & F. Barros et al. 3200 (SP) & \\
\hline E. sclerophyllum Lindl. & G.A. Damasceno Junior et al. 3433 (COR, SP) & \\
\hline Erycina pusilla. (L.) N.H.Williams \& M.W.Chase & Ostetto (2015) & \\
\hline Eulophia alta (L.) Fawc. \& Rendle & A. Pott et al. 8695 (HMS & \\
\hline Eurystyles cf. cotyledon Wawra & V.B. Paiva Neto 53 (SP) & NMS \\
\hline Galeandra beyrichii Rchb. f. & F.C. Hoehne CLTEMT-AM 4113 (R) & Holótipo de Galeandra coxinensis Hoehne \\
\hline G. blanchetii E.S.Rand & V.B. Paiva Neto 12 (SP) & NMS \\
\hline G. montana Barb.Rodr. & G. Hatschbach 23753 (NY, UPCB) & \\
\hline G. paraguayensis Cogn. & Ostetto (2015) & \\
\hline G. styllomisantha (Vell.) Hoehne & A. Pott \& V.J. Pott 11171 (CEN, CPAP, HMS) & \\
\hline Galeottia ciliata (C.Morel) Dressler \& Christenson & V.B. Paiva Neto 03 (SP) & \\
\hline Gomesa recurva R.Br. & Ostetto (2015) & \\
\hline Habenaria amambayensis Schltr. & T.M. Pedersen 12256 (HB, MBM) & \\
\hline
\end{tabular}


Quadro 1. Cont.

\begin{tabular}{|c|c|c|}
\hline Espécie & Voucher ou Referência Bibliográfica & Observações \\
\hline H. anisitsii Kraenzl. & B. Orssich s.n. (HB) & NMS \\
\hline H. aricaensis Hoehne & A. Pott 5530 (CPAP, SP) & \\
\hline H. bractescens Lindl. & L. Cullen s.n. (HB, ICN, PACA, RB, SP) & \\
\hline H. ekmaniana Kraenzl. & G. Hatschbach 26125 (MBM, HB) & \\
\hline H. floribunda Lindl. & V.B. Paiva Neto 75 (SP) & NMS \\
\hline H. glazioviana Kraenzl. ex Cogn. & A. Pott et al. 5591 (CPAP, MBM, SP) & \\
\hline H. heptadactyla Rchb.f. & A. Pott \& V.J. Pott 6767 (CPAP) & \\
\hline H. johannensis Barb.Rodr. & G. Hatschbach 26101 (MBM, HB, PCB) & \\
\hline H. juruenensis Hoehne & I.M. Bortolotto et al. 175 (COR, SP) & \\
\hline H. longicauda Hook. & G.F. Arbocz 7141 (SP) & NMS \\
\hline H. cf. nuda Lindl. & S.N. Moreira et al. 324 (CGMS) & \\
\hline H. obtusa Lindl. & E.P. Heringer et al. 769 (MG, IBGE) & \\
\hline H. orchiocalcar Hoehne & A. Pott 2531 (CEN, CPAP, SP, UB) & \\
\hline H. petalodes Lindl. & A. Pott \& V.J. Pott 15020 (CGMS) & \\
\hline H. polycarpa Hoehne & M. Pereira \& R.A. Mauro 04 (CPAP, MBM) & \\
\hline H. pungens Cogn. ex Kuntze & S.N. Moreira et al. 323 (CEN, CGMS) & NMS \\
\hline H. regnellii Cogn. & F. Chagas e Silva 109A (IBGE) & NMS \\
\hline H. repens Nutt. & A. Pott et al. 2756 (CPAP, SP) & \\
\hline H. schwackei Barb. Rodr. & G. Hatschbach 26061 (MBM, HB) & NMS \\
\hline H. tamanduensis Schltr. & G. Hatschbach 45884 (MBM) & NMS \\
\hline Heterotaxis equitans (Schltr.) Ojeda \& Carnevali & Cribb (1998) & Citada como Maxillaria equitans (Schltr.) Garay \\
\hline $\begin{array}{l}\text { Houlletia odoratissima Linden ex Lindl. \& } \\
\text { Paxton }\end{array}$ & V.B. Paiva Neto 27 (SP) & \\
\hline Ionopsis utricularioides (Sw.) Lindl. & V.J. Pott et al. 4855 (CEN, CPAP, HMS) & \\
\hline Isochilus linearis (Jacq.) R.Br. & D.P. Rodriguez 10 (COR, SP) & \\
\hline Koellensteinia eburnea (Barb.Rodr.) Schltr. & G. Hatschbach 35979 (MBM) & \\
\hline Laelia gloriosa (Rchb. f.) L.O.Williams & M.A. Lima ex F. Barros 3192 (SP) & $\begin{array}{l}\text { Citada por Ostetto (2015) como } \\
\text { Schomburgkia crispa Lindl. }\end{array}$ \\
\hline Lankesterella ceracifolia Barb.Rodr. & Ostetto (2015) & \\
\hline Leptotes unicolor Barb. Rodr. & Rech et al. (2011) & \\
\hline Liparis cogniauxiana F.Barros \& L.Guimarães & Cribb (1998) & \\
\hline L. nervosa (Thunb.) Lindl. & V.B. Paiva Neto 52 (SP) & \\
\hline Lockhartia goyazensis Rchb. f. & F. Barros 1163 (SP) & NMS \\
\hline L. imbricata (Lam.) Hoehne & V.B. Paiva Neto 94 (SP) & NMS \\
\hline L. ludibunda Rchb.f. & Ostetto (2015) & \\
\hline Lophiaris morenoi (Dodson \& Luer) Braem & G.A. Damasceno Junior et al. 2031 (COR, SP) & \\
\hline L. nana (Lindl.) Braem & V.B. Paiva Neto 108 (SP) & \\
\hline L. pumila (Lindl.) Braem & $\begin{array}{l}\text { Bochese et al. (2011), Rech et al. (2011), Ostetto } \\
\text { (2015) }\end{array}$ & \\
\hline Macradenia multiflora (Kraenzl.) Cogn. & H. D. Bicalho s.n. (SP175143) & \\
\hline Macroclinium mirabile (C.Schweinf.) Dodson & Ostetto (2015) & \\
\hline Mesadenella cuspidata (Lindl.) Garay & V.B. Paiva Neto \& R. Antonelli 79 (SP) & \\
\hline $\begin{array}{l}\text { Microchilus arietinus (Rchb.f. \& Warm.) } \\
\text { Ormerod }\end{array}$ & V.B. Paiva Neto 30 (SP) & NMS \\
\hline $\begin{array}{l}\text { Microlaelia lundii (Rchb.f.) Chiron \& } \\
\text { V.P.Castro }\end{array}$ & Rech et al. (2011) & $\begin{array}{c}\text { Citada como Laelia lundii } \\
\text { (Rchb. f. \& Warm.) Rchb. f. \& Warm. }\end{array}$ \\
\hline Miltonia flavescens Lindl. & M.M. Barreto s.n. (ESA6834) & \\
\hline M. regnellii Rchb.f. & Ostetto (2015) & \\
\hline Mormodes auriculata F.E.L.Miranda & F. Miranda $960(\mathrm{RB})$ & Holótipo \\
\hline Myoxanthus lonchophyllus (Barb.Rodr.) Luer & F. Barros et al. 3189 (SP) & NMS \\
\hline Notylia longispicata Hoehne \& Schltr. & Ostetto (2015) & \\
\hline N. lyrata Sp. Moore & V.B. Paiva Neto 43 (SP) & \\
\hline N. microchila Cogn. & Ostetto (2015) & \\
\hline Octomeria warmingii Rchb.f. & $\begin{array}{l}\text { M.A.C. Chaparro \& I.M. Bortolotto } 10 \text { (COR, } \\
\text { CPAP, SP) }\end{array}$ & \\
\hline Oeceoclades maculata (Lindl.) Lindl. & D.P. Rodriguez 61 (COR, SP) & \\
\hline Orleanesia yauaperyensis Barb.Rodr. & V.B. Paiva Neto 37 (SP) & NMS \\
\hline
\end{tabular}


Quadro 1. Cont.

\begin{tabular}{|c|c|c|}
\hline Espécie & Voucher ou Referência Bibliográfica & Observações \\
\hline Ornithocephalus cujeticola Barb.Rodr. & V.B. Paiva Neto 69 (SP) & NMS \\
\hline $\begin{array}{l}\text { Pabstiella aff. leucosepala (Loefgr.) F.Barros } \\
\& \text { C.F.Hall }\end{array}$ & V.B. Paiva Neto 54 (SP) & \\
\hline P. tricolor (Barb.Rodr.) Luer & Cribb (1998) & $\begin{array}{c}\text { Citada como Pleurothallis tricolor } \\
\text { (Barb. Rodr.) Cogn. }\end{array}$ \\
\hline P. tripterantha (Rchb.f.) F.Barros & V.B. Paiva Neto 90SP) & \\
\hline Palmorchis sp. & S. Aragaki \& U.M. Resende 935 (CGMS, COR) & \\
\hline Pelexia pterigantha (Rchb.f. \& Warm.) Schltr. & V.B. Paiva Neto \& R. Antonelli 73 (SP) & \\
\hline Phragmipedium vittatum (Vell.) Rolfe & V.J. Pott \& A. Pott 9707 (CGMS) & \\
\hline Platythelys schlechterana (Hoehne) Garay & A. Pott et al. 2757 (CPAP, SP) & NMS \\
\hline Plectrophora edwallii Cogn. & Cribb (1998) & \\
\hline Polystachya concreta (Jacq.) Garay \& Sweet & G.A. Damasceno Junior et al. 1194 (CGMS, SP) & \\
\hline P. estrellensis Rchb.f. & J. Correa_Gomes 1949 (SP) & NMS \\
\hline P. foliosa (Hook.) Rchb.f.. & Ostetto (2015) & \\
\hline P. micrantha Schltr. & V.B. Paiva Neto 10 (SP) & NMS \\
\hline Ponthieva montana Lindl. & F. Barros et al. 3170 (SP) & NMS \\
\hline Prescottia densiflora (Brongn.) Lindl. & Ostetto (2015) & \\
\hline P. oligantha (Sw.) Lindl. & A.E.H. Salles 95 (IBGE) & \\
\hline Prosthechea fragrans (Sw.) W.E.Higgins & Ostetto (2015) & $\begin{array}{l}\text { Citada como Anacheillium fragrans } \\
\text { (Sw.) Acuña }\end{array}$ \\
\hline P. sessiliflora (Edwall) W.E.Higgins & V.B. Paiva Neto 18 (SP) & NMS \\
\hline P. vespa (Vell.) W.E.Higgins & V.B. Paiva Neto 04 (SP) & \\
\hline Pteroglossa macrantha (Rchb.f.) Schltr. & Ostetto (2015) & \\
\hline Rodriguezia decora (Lem.) Rchb.f.. & Ostetto (2015) & \\
\hline Sacoila lanceolata (Aubl.) Garay & A. Pott 3849 (CPAP) & \\
\hline Sarcoglottis acaulis (J.E.Sm.) Schltr. & V.B. Paiva Neto 42 (SP) & NMS \\
\hline S. curvisepala Szlach. \& Rutk. & G.A. Damasceno Junior 1965 (CGMS, COR, SP) & \\
\hline S. aff. fasciculata (Vell.) Schltr. & V.J. Pott et al. 226 (CPAP) & \\
\hline S. heringeri Pabst & Ostetto (2015) & \\
\hline S. schwackei (Cogn.) Schltr. & Ostetto (2015) & \\
\hline S. uliginosa Barb.Rodr. & V.J. Pott \& A. Pott 9706 (CGMS) & \\
\hline Sauroglossum nitidum (Vell.) Schltr. & V.B. Paiva Neto 91 (SP) & NMS \\
\hline Scaphyglottis livida (Lindl.) Schltr. & F. Barros et al. 3194 (SP) & \\
\hline S. modesta (Rchb.f.) Schltr. & Ostetto (2015) & \\
\hline S. prolifera (R. Br.) Cogn. & V.B. Paiva Neto 78 (SP) & NMS \\
\hline S. stellata Lodd. ex Lindl. & Ostetto (2015) & \\
\hline Scuticaria sp. & M.A. Chaparro et al. s.n. (COR3602) & \\
\hline Sobralia sp. & Ostetto (2015) & \\
\hline Solenidium lunatum (Lindl.) Schltr. & Ostetto (2015) & \\
\hline Sophronitis cernua Lindl. & D.P. Rodriguez 5 (COR, SP) & \\
\hline Specklinia grobyi (Batem. ex Lindl.) F.Barros & Ostetto (2015) & \\
\hline Trichocentrum albo-coccineum Lindl. & V.B. Paiva Neto 16 (SP) & \\
\hline T. fuscum Lindl. & Ostetto (2015) & \\
\hline Trichopilia brasiliensis Cogn. & V.B. Paiva Neto 76 (SP) & \\
\hline Trigonidium tenue Lodd. & V.B. Paiva Neto 26 (SP) & \\
\hline Vanilla chamissonis Klotzsch & M.A. Lima 06 (SP) & \\
\hline V. edwallii Hoehne & Ostetto (2015) & \\
\hline V. lindmaniana Kraenzl. & Cribb (1998) & \\
\hline V. odorata C. Presl & V.B. Paiva Neto \& M.A. Lima 47 (SP) & NMS \\
\hline V. palmarum (Salzm. ex Lindl.) Lindl. & D.P. Rodriguez \& H. Baracat 60 (COR, SP) & \\
\hline V. planifolia Jacks. ex Andrews & Ostetto (2015) & \\
\hline V. pompona Schiede & Ostetto (2015) & \\
\hline V. ribeiroi Hoehne & Ostetto (2015) & \\
\hline Warmingia eugenii Rchb.f. & Ostetto (2015) & \\
\hline Xylobium foveatum (Lindl.) Nichols & D.P. Rodriguez 6 (COR, SP) & \\
\hline$X$. variegatum (Ruiz \& Pav.) Mansf. & M.A. Lima 07 (SP) & \\
\hline Zygopetalum maxillare Lodd. & Ostetto (2015) & \\
\hline Zygostates alleniana Kraenz. & Ostetto (2015) & \\
\hline
\end{tabular}




\section{REFERÊNCIAS}

Atwood, J.T. 1986. The size of the Orchidaceae and the systematic distribution of epiphytic orchids. Selbyana 9: 171-186.

Barros, F., Vinhos, F., Rodrigues, V.T., Barberena, F.F.V.A., Fraga, C.N., Pessoa, E.M, Forster, W., Menini Neto, L., Furtado, S.G., Nardy, C., Azevedo, C.O. \& Guimarães, L.R.S. 2015. Orchidaceae In Lista de Espécies da Flora do Brasil. Jardim Botânico do Rio de Janeiro, Rio de Janeiro. Disponível em: http://floradobrasil.jbrj.gov.br/jabot/ floradobrasil/FB179. Acessado em 14.09.2015.

Bocchese, R.A., Costacurta, M.B. \& Souza, W. 2011. Ocorrência de orquídeas nativas em trechos de mata ciliar do Rio Sucuriu, Município de Costa Rica - MS. In Anais do Encontro sobre Orquídeas Nativas e Adaptadas ao Cerrado Brasileiro (V.B. Paiva Neto, ed.). Universidade Federal do Mato Grosso do Sul, Chapadão do Sul, p. 43-46.

Bocchese, R.A. 2015. Orquídeas - MS: Espécies nativas do Mato Grosso do Sul. Disponível em: http://orquideas-ms.blogspot.com.br/2012/06/ dendrobium-floribundum.html. Acessado em 14.02015.

Boldrini, I.I., Trevisan, R. \& Schneider, A.A. 2008. Estudo florístico e fitossociológico de uma área às margens da lagoa do armazém, Osório, Rio Grande do Sul, Brasil. Revista Brasileira de Biociências 6(4): 355-367.

Chase, M.W., Cameron, K.M., Barret, R.L. \& Freudenstein, J.V. 2003. DNA data and Orchidaceae systematics: a new phylogenetic classification. In Orchid conservation (K.W. Dixon, S.P. Kell, R.L. Barrett \& P.J. Cribb, eds.). Natural History Publications, Sabah. p. $69-89$.

Cribb, P. 1998. Orchidaceae. In Prodromus Florae Matogrossensis. Part I. Checklist of Angiosperms (B. Dubs, ed.). Betrona-Verlag, Küsnacht, p. 221-230.

Dubs, B. 1998. Prodromus Florae Matogrossensis. Part I. Checklist of Angiosperms. Betrona-Verlag, Küsnacht.

Gomes-Klein, V.L. \& Rizzo, J.A. 2006. Estudos sobre a flora do centrooeste do Brasil: situação atual e perspectivas. In Os avanços da botânica no início do século XXI (J.E.A. Mariath \& R.P. Santos, ed.). Sociedade Botânica do Brasil, Porto Alegre, p. 670-675.

Hoehne, F.C. 1951. Índice bibliográfico e numérico das plantas colhidas pela Comissão Rondon. Instituto de Botânica, São Paulo.

Mato Grosso do Sul. 2009. Dados Estatísticos de Mato Grosso do Sul 2009. Secretaria de Estado de Meio Ambiente, do Planejamento, da Ciência e Tecnologia, Governo do Estado do Mato Grosso do Sul, Campo Grande.

Ministério do Meio Ambiente. 2012. Mapas de cobertura vegetal dos biomas brasileiros. Disponível em: http://mapas.mma.gov.br/mapas/ aplic/probio/datadownload.htm. Acessado em 30.08.2012.

Munhoz, C.B.R. \& Felfili, J.M. 2006. Fitossociologia do estrato herbáceosubarbustivo de uma área de campo sujo no Distrito Federal, Brasil. Acta botanica brasilica 20(3): 671-685.
2008. Fitossociologia do estrato herbáceo-subarbustivo em campo limpo úmido no Brasil Central. Acta botanica brasilica 22(4): 905-913.

Ostetto, S. 2015. Orquídeas de Mato Grosso do Sul. Gráfica e Editora Alvorada, Campo Grande.

Pabst, G.F.J. \& Dungs, F. 1975. Orchidaceae Brasilienses, vol. 1. Kurt Schmersow, Hisdesheim.

. 1977. Orchidaceae Brasilienses, vol. 2. Kurt Schmersow, Hisdesheim.

Pagotto, T.C.S. \& Souza, P.R. (orgs.). 2006. Biodiversidade do Complexo Aporé-Sucuriú : subsídios à conservação e ao manejo do Cerrado : área prioritária 316-Jauru. Universidade Federal do Mato Grosso do Sul, Campo Grande.

Paiva Neto, V.B., Lima Junior, M.A., Barros, F., Lima, S.F., Zuffo, M.C.R., Boaretto, A.G., Fernandes, M.A. \& Oliveira, J.F. 2011 a. Dados preliminares de levantamento florístico de espécies da família Orchidaceae em Chapadão do Sul - MS. In Anais do Encontro sobre Orquídeas Nativas e Adaptadas ao Cerrado Brasileiro (V.B. Paiva Neto, ed.). Universidade Federal do Mato Grosso do Sul, Chapadão do Sul, p. 14-17.

Paiva Neto, V.B., Lima Junior, M.A., Barros, F., Lima, S.F., Zuffo, M.C.R., Boaretto, A.G., Fernandes, M.A. \& Zandonadi, G.F. 2011b. Dados preliminares de levantamento florístico de espécies da família Orchidaceae em Costa Rica - MS In Anais do Encontro sobre Orquídeas Nativas e Adaptadas ao Cerrado Brasileiro (V.B. Paiva Neto, ed.). Universidade Federal do Mato Grosso do Sul, Chapadão do Sul, p. 18-21.

Palma, C.B., Inácio, C.D., Jarenkow, J.A. 2008. Florística e estrutura da sinúsia herbácea terrícola de uma floresta estacional de encosta no Parque Estadual de Itapuã, Viamão, Rio Grande do Sul, Brasil. Revista Brasileira de Biociências 6(3): 151-158.

Pellizzaro, K.F., Batista, J.A.N. \& Bianchetti, L.B. 2004. O gênero Oncidium Sw. (Orchidaceae) no Distrito Federal, Brasil. Boletim do Herbário Ezequias Paulo Heringer 14: 128-143.

Rech, A.R., Rosa, Y.B.C.J. \& Rosa Junior, E.J. 2011. Levantamento e características ecológicas de Orchidaceae da mata ciliar do Rio Dourados, Dourados - MS. Revista Árvore 35(3, suppl. 1): 717-724.

Rodrigues, D.P., Barros, F., Damasceno Júnior \& Bortolotto, I.M. 2009. Levantamento da família Orchidaceae no Morro Santa Cruz, Municípios de Corumbá e Ladário, Mato Grosso do Sul, Brasil. Hoehnea 36(4): 613-636.

SpeciesLink. 2012. Disponível em: http://www.splink.org.br/ index?lang=pt. Acessado em 20.08.2012.

Thiers, B. 2012 [continuously updated]. Index Herbariorum: A global directory of public herbaria and associated staff. New York Botanical Garden's Virtual Herbarium. New York Boanical Garden, New York. Disponível em: http://sweetgum.nybg.org/ih/. Acessado em 30.08.2012. 